Sharif University of Technology
Scientia Iranica
SCIENTIA

\title{
Submicron particle deposition in pulmonary alveoli during cyclic breathing
}

\author{
M. Monjezi ${ }^{a}$, M.S. Saidi ${ }^{a}, *$ and G. Ahmadi ${ }^{b}$ \\ a. School of Mechanical Engineering, Sharif University of Technology, Tehran, Iran. \\ b. Department of Mechanical and Aeronautical Engineering, Clarkson University, Potsdam, New York, USA. \\ Received 27 August 2015; received in revised form 4 May 2016; accepted 13 August 2016
}

\section{KEYWORDS}

FSI;

Particle deposition;

Cyclic breathing;

Diffusion.

\begin{abstract}
The prediction of deposition efficiency of submicron particles in the pulmonary alveoli has received special attention due to its importance for drug delivery systems and for assessing air pollutants health risks. In this work, the pulmonary alveoli of a healthy human are idealized by a three-dimensional honeycomb-like configuration and a fluid-structure interaction analysis is performed. In contrast to previous works in which the inlet flow rate is predefined, in this model, a negative pressure is imposed on the outside surface of the alveolus which causes air to flow in and out of the alveolus. The resulting flow patterns confirmed that there was no circulation in the terminal alveolus. The predicted alveolar airflow was used to calculate the trajectories of submicron particles using the Lagrangian approach. Our findings suggest that an accurate simulation requires including at least ten breathing cycles, considering a parabolic radial distribution of injected particles and continuous injection. The presented results show high deposition efficiency for submicron sizes in the alveolar region if these particles can reach the alveolar region. Therefore, the vesicles technology in which particle agglomerates would be released after the vesicle reaching the alveoli is suggested for targeted drug delivery to the alveolar region.
\end{abstract}

(C) 2017 Sharif University of Technology. All rights reserved.

\section{Introduction}

Determination of airflow and particle deposition in the alveolar region has been of considerable interest to many researchers due to its importance in pulmonary drug delivery systems and/or the adverse health effects of particulate air pollutants. The huge surface area of alveoli with slow metabolism compared to liver or gastrointestinal track makes it a desirable target for aerosolized drug delivery. For example, $\alpha 1-$ antitypsin [1], inhaled insulin or immunoglobulin [2], and nebulized morphine [3] have been successfully administered. New drug-targeting strategies have also been developed for delivering nanoparticles via

\footnotetext{
*. Corresponding author. Tel.: +982166165558;

Fax: +982166000021

E-mail address: mssaidi@sharif.edu (M.S. Saidi)
}

some carriers which dissolve immediately after contact with the alveolar aqueous region for therapeutic or diagnostic purposes [4]. On the other hand, exposure to particulate air pollutants causes pulmonary diseases and toxicity effects [5]. Hofmann [6] and Hussain et al. [7] presented a review on particle deposition in the whole lung models. There also have been a number of studies focusing on deposition efficiency in alveoli. For example, Haber et al. [8] used a spherical model of rhythmically expanding and contracting alveolus and approximated the gravitational deposition of particles using a mathematical framework. Harrington et al. [9] also used an axisymmetric duct model with rigid walls in order to simulate deposition of $1-5 \mu \mathrm{m}$ particles. Later, they included duct expansion in their model [10] and concluded that it enhanced the deposition of the particles of these sizes. Ma and Darqueene [11] predicted the deposition efficiency of 1 and $3 \mu \mathrm{m}$ parti- 
cles under cyclic breathing using a three-dimensional model for acinar airways. They reported $33 \%$ and $75 \%$ deposition efficiency, respectively, for 1 and $3 \mu \mathrm{m}$ aerosols under normal gravity condition. Henry et al. [12] reported flow mixing mechanism in rhythmically expanding idealized spherical models of alveolus for submicron particles. In these studies, a sinusoidal function of time was used for expansion and contraction of alveolus, where it was assumed that the model deformed isothropically. Dailey and Ghadiali [13] performed a Fluid-Solid-Interaction (FSI) analysis on a two-dimensional alveolus model to find microparticle transport in alveoli. They also used a sinusoidal form of pressure load. The FSI simulation generates a more realistic alveolus wall deformation that leads to transient airflow fields through the coupled fluidstructure interactions. One common assumption in these earlier studies has been that all particles are inhaled spontaneously at the start of inhalation, while, under the normal breathing conditions, particles are continuously carried by the inhaled air.

In this work, a Flow-Structure Interaction (FSI) model is used to study the normal breathing airflow in a three-dimensional model of alveoli. For the mechanical properties of alveolar tissue, a more realistic model based on the earlier experimentally reported data is used. In addition, a more physiologically realistic boundary condition compared to the earlier literature is employed. First, the characteristic of experimental spirometer profile is used to find the negative pressure load on the alveolar. Second, particles are injected during inhalation with a transient concentration profile similar to the inlet airflow. The presented model provides a potential computational tool for predicting particle deposition efficiency incorporating physiologically realistic alveolar wall motion.

The remainder of the article is organized in the following sections. Section 2 provides the numerical methodology including the geometry, governing equations, boundary conditions, FSI simulation, and particle tracking approach. Section 3 presents the simulation results for fluid, solid, and particle dynamics. Discussion and limitations are provided in Section 4. Finally, a summary of the results and conclusions is presented in Section 5.

\section{Methodology}

\subsection{Geometry}

In order to represent one terminal alveolus, a honeycomb-like configuration, which uses a cluster of truncated octahedrons to obtain nearly space-filling polyhedral elements, is used [14]. Figure 1 shows the three-dimensional geometry of the alveolus with rounded corners. The hexagonal side and total length of the alveolus are, respectively, $80 \mu \mathrm{m}$ and $1000 \mu \mathrm{m}$.

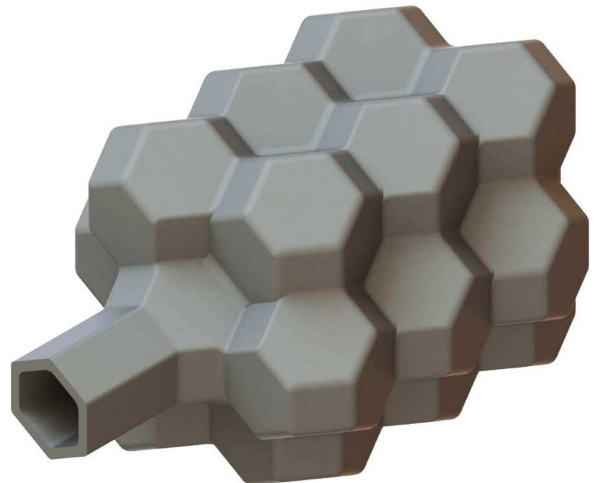

Figure 1. 3D alveolar sac geometry with a single entrance.

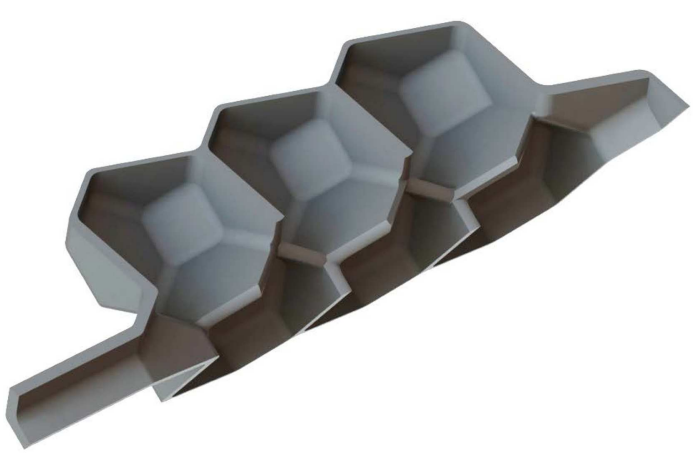

Figure 2. The one-sixth geometry used in the computational model.

The model can be divided into six similar parts using symmetry planes. Therefore, the computational model is confined to one-sixth of this configuration that is shown in Figure 2. The thickness of alveolar wall tissue is considered to be $8 \mu \mathrm{m}$ for a healthy human subject [15].

\subsection{Fluid domain}

The governing equations are the continuity and the Navier-Stokes for an incompressible fluid. They are:

$$
\begin{aligned}
& \frac{\partial v_{i}}{\partial x_{i}}=0 \\
& \rho_{f} \frac{\partial v_{i}}{\partial t}+\rho_{f} v_{i} \frac{\partial v_{i}}{\partial x_{j}}=-\frac{\partial p}{\partial x_{i}}+\mu \frac{\partial^{2} v_{i}}{\partial x_{j} \partial x_{j}},
\end{aligned}
$$

where $\rho_{f}, \mu, v_{i}, x_{i}, t$, and $p$ represent air density, viscosity, velocity vector, position vector, time, and pressure, respectively.

\subsection{Structural domain}

The equation of balance of momentum equation for the solid alveolar wall is given as:

$$
\rho_{s} \frac{\partial^{2} d_{i}^{s}}{\partial t^{2}}=\frac{\partial \sigma_{i j}^{s}}{\partial x_{j}}
$$


where $\rho_{s}=1050 \mathrm{~kg} / \mathrm{m}^{3}$ is the tissue density, and $d_{i}^{s}$ and $\sigma_{i j}^{s}$ stand for the solid displacement and Cauchy stress tensor, respectively.

For the tissue material, Sugihara et al. [15] used their experimental data to fit a semi-empirical model. Accordingly, the stress-strain law for alveolar tissue is given by:

$$
\sigma=\lambda^{8}-\lambda^{6.4} \quad(\mathrm{kPa})
$$

where $\lambda$ is the stretch ratio of alveolar tissue. We have incorporated viscoelastic properties of alveolar connective tissue in a general Mooney-Rivlin hyperelastic material model. The corresponding reduced relaxation function for viscoelasticity of alveolar wall tissue is given by [16]:

$$
G(t)=0.4+0.6(t+1)^{-0.5} \text {. }
$$

\subsection{Fluid-structure interaction implementation}

The FSI simulation is performed in ADINA 8.8 software. Tetrahedral elements are used for both fluid and solid domains. The fluid and solid primary meshes are comprised of 1,273,000 and 533,000 elements, respectively. Sample surface meshes are shown in Figure 3.

\subsection{Boundary conditions for FSI simulation}

The driving parameter in alveolar expansion and contraction is the transmural pressure. For normal breathing, the pressure-volume relationship is approximately linear. Therefore, the form of transmural pressure variation can be obtained by integrating the spirometer flow profile [17]. Since this study is focused on a terminal alveolus, not the whole alveoli, we should scale this achieved curve by a decrease factor in a way that a physiological value for alveolus volume change can be obtained. The scaled pressure profile for the boundary condition is shown in Figure 4.

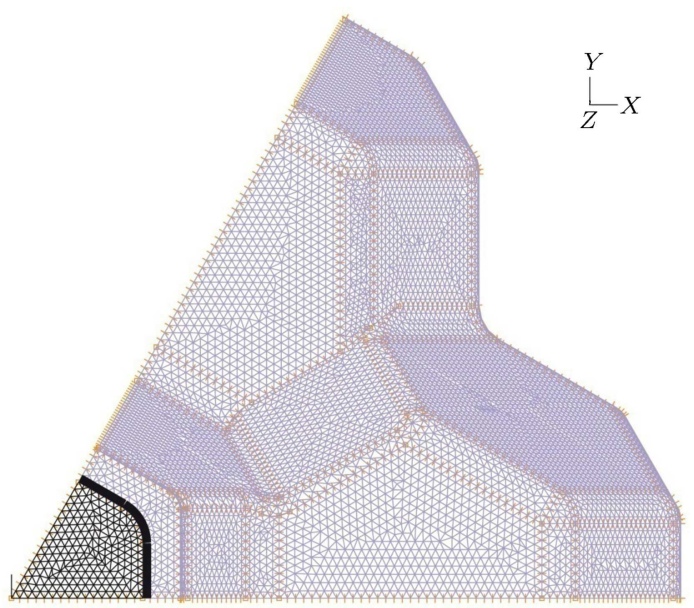

Figure 3. Sample surface mesh of solid and fluid regions.

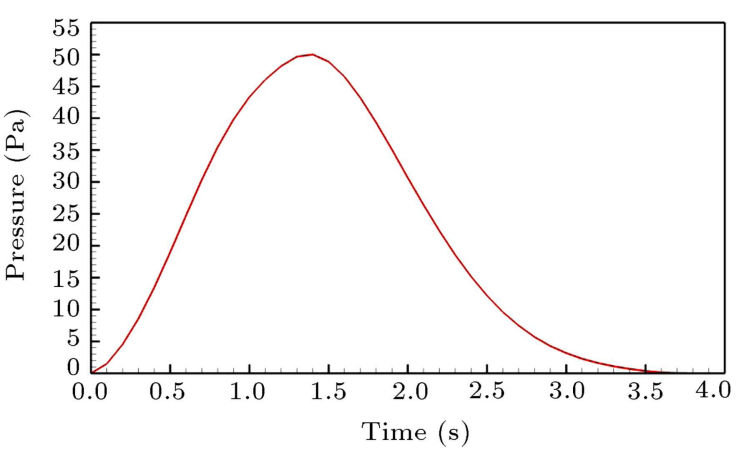

Figure 4. Pressure profile boundary condition.

At the interface of fluid and solid domains, two boundary conditions are applied. These conditions include continuity of displacement and tractions, which are:

$$
\begin{aligned}
& d_{i}^{f}=d_{i}^{s}, \\
& n_{j} \sigma_{i j}^{f}=n_{j} \sigma_{i j}^{s},
\end{aligned}
$$

where:

$$
\sigma_{i j}^{f}=-p \delta_{i j}+\mu\left(\frac{\partial v_{i}}{\partial x_{j}}+\frac{\partial v_{j}}{\partial x_{i}}\right) .
$$

In these equations, $n_{j}$ represents the interface normal vector, $\delta_{i j}$ is the Kronecker delta function, $d_{i}^{f}$, $d_{i}^{s}, \sigma_{i j}^{f}$ and $\sigma_{i j}^{s}$ stand for the fluid and solid nodal displacements, and the fluid and solid stress tensors, respectively.

The symmetry condition is also applied on the two symmetry planes in both fluid and solid domains. For the inlet boundary in fluid domain, a reference pressure is set.

\subsection{Particle tracking}

In this study, for a dilute suspension of submicron-size particles, a one-way couple assumption is used. That is, the airflow carries the particles but the motion of these small particles does not influence the airflow field. In addition, there are no particle-particle interactions. Since airflow is decoupled from the particle motions, the FSI simulation is first performed and the transient flow fields for ten successive breathing cycles are evaluated. Then, the fluid flow solution and the mesh motion are exported from the FSI simulation and are used in an in-house code written in $\mathrm{C}^{++}$for calculation of particle tracking. A large number of particles are then injected at the inlet of the alveolus and tracked during several breathing cycles. These particles represent the fraction of particles inhaled at the trachea and then penetrated through the airway tree to the alveolar region. The inhouse code integrates the particle equation of particle motion given as:

$$
\frac{d u_{i}^{p}}{d t}=\beta\left(u_{i}^{f}-u_{i}^{p}\right)+n(t)+g_{i}
$$




$$
\begin{aligned}
& n(t)=G_{i} \sqrt{\frac{\pi S_{0}}{\Delta t}}, \\
& S_{0}=\frac{2 k T \beta}{\pi m}, \\
& \tau_{R}=\frac{\rho_{p} d_{p}^{2} C_{c}}{18 \mu} .
\end{aligned}
$$

In this equation, $\beta$ is the reverse of the particle relaxation time, $n(t)$ stands for the Brownian force, $G_{i}$ is a Gaussian random number, $C_{c}$ is the Cunningham slip correction factor, and $S_{0}$ is the spectral intensity of Brown excitation [18]. Here, $u_{i}^{f}, u_{i}^{p}$, and $g_{i}$, respectively, represent fluid velocity, particle velocity, and the acceleration of gravity. Note that since particle density is much larger than that of the air, the buoyancy and added mass forces that are negligibly small are neglected.

A particle is considered deposited if it touches the wall. That is, if the center of particle reaches a distance from a wall that is equal to its radius or smaller.

The time step for integration of particle equation is determined based on the particle relaxation time and the time needed for a particle to travel a grid cell. Since the particle relaxation time for the submicron particles is much smaller than the time for traveling a grid cell, a fraction of particle relaxation time is used for integration time step. Sensitivity analysis is also performed by using smaller time steps and checking whether the results have changed. It is concluded that $10^{-8} \mathrm{sec}$ for 0.1 -micron particles and $5 \times 10^{-8} \mathrm{sec}$ for 1-micron particles are sufficiently small.

In contrast to similar earlier studies [9], in which all particles were injected at the start of inspiration phase, a more physiologically realistic condition via transient injection is used in the present study. The transient profile of inlet airflow is used to find the transient profile for particle injection. The normalized values of inlet flow profile are obtained in $0.1 \mathrm{~s}$ time increments over the inhalation time. The initial velocity of the particles is set to that of the local airflow velocity. For the radial profile of injected particles, we have used the findings of Tsuda et al. [19], who reported that particles initially introduced with a uniform radial concentration became fully developed in the alveolar ducts with a parabolic profile. The maximum of this fully developed profile is 2.5 times its mean. The uniform distribution of injected particles is turned into a parabolic distribution by applying a weighting coefficient for a number of particles injected from each of uniformly distributed locations based on the distance from the wall. Thus, the number of particles injected from the center would be larger than those injected near the wall. 67 locations uniformly distributed on $1 / 6$ of the alveolus inlet as shown in Figure 5 are used to inject particles in each time

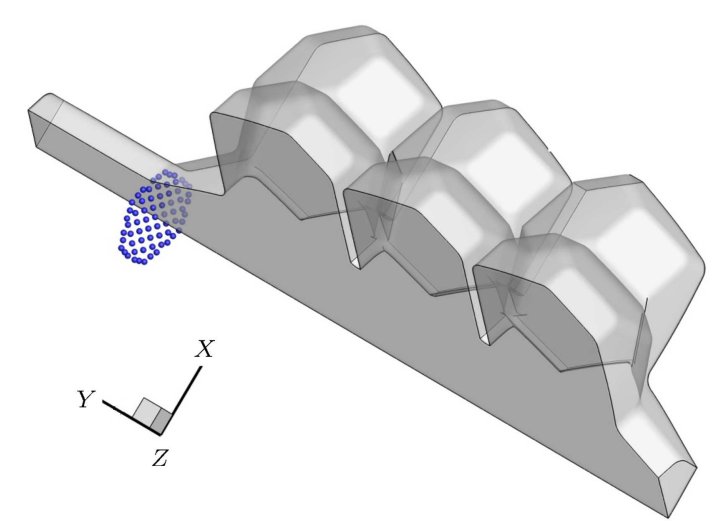

Figure 5. Locations of particle injection.

interval. In each time step, the injected particles move randomly around their primary locations to cover the whole inlet surface. New particles are added during inspiration in each breath and simulation continues to reach a quasi-steady condition. By this approach, the suspended particles that have not sufficient time to deposit on the walls of alveoli may deposit during the next breathing cycles. After about ten cycles, the fraction of deposited particles would be stable.

In this study, we are interested in the influence of particle aerodynamic diameter on their deposition efficiency. First, the case of particle transport and deposition under microgravity environment, where sedimentation effect is negligible, is simulated. Then, in order to elucidate the sedimentation effect, the gravity term is added to the governing equations and the transport and deposition under normal gravity are studied.

Similar to the approach of Ma and Darqueene [11], six different orientations for the alveolus with respect to gravity are considered and, due to axisymmetric nature of the alveolus, only the up [(positive $Z(Z p)]$, down [negative $Z(Z n)$ ], and right [positive $Y(Y p)$ ] gravity orientations are simulated. The average deposition efficiency is approximated by the weighted average for three independent orientations. Accordingly, the average deposition efficiency for alveolus is $(Z p+Z n+$ $4 Y p) / 6$.

\section{Results}

\subsection{Fluid and solid dynamics}

Our fluid results show that cyclic wall motion induced by change in transmural pressure causes airflows in and out of the alveolus. At the entrance of alveolus, both Womersley number and Reynolds number are smaller than 0.1. Therefore, the creeping laminar fluid flow characteristics are expected. In Figure 6, velocity contours and streamlines at $t=0.6 \mathrm{~s}$, which is the instant of maximum inhalation, are shown. The pick velocity magnitude at this time is $2.8 \mathrm{~mm} / \mathrm{s}$. This 


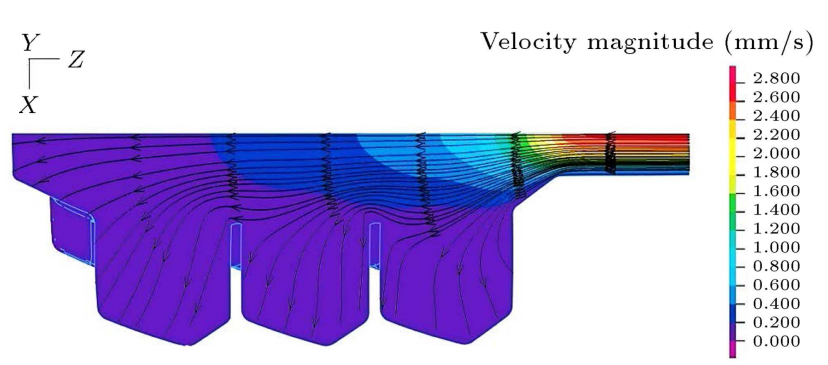

Figure 6. Velocity magnitude contours and streamlines in alveolus at $t=0.6 \mathrm{~s}$.

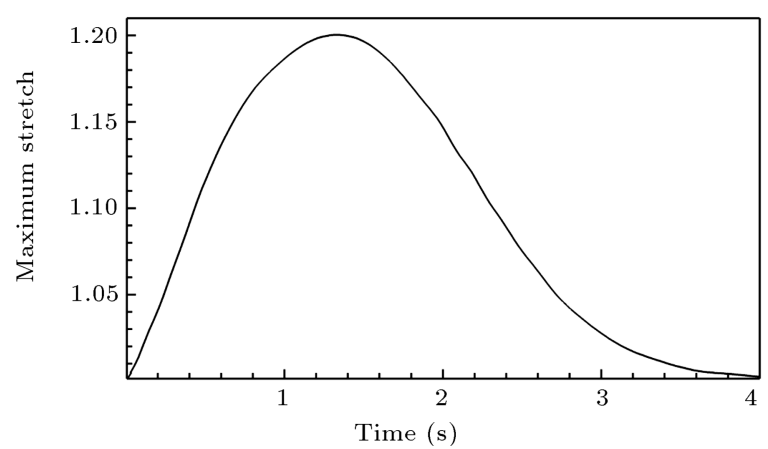

Figure 7. Maximum local stretch in alveolus at different times.

figure confirms the presence of laminar radial flow at the terminal alveolus with no recirculation zone, which is in agreement with the findings of earlier studies $[17,20,21]$.

The maximum local stretch at different times is shown in Figure 7 . The maximum value for local stretch is about 1.2 occurring at $t=1.4$, which is in agreement with the X-ray microscopy performed by Raush et al. [22]. We have presented more results about alveolus mechanics elsewhere [23].

\subsection{Particle deposition for zero-gravity condition}

3.2.1. Deposition during successive breathing cycle Deposition efficiencies of particles with different sizes during successive breathing cycles are evaluated. Particles are introduced at the inlet of alveolus and their trajectories are tracked. The deposition efficiency in the $N$ th cycle is defined as the ratio of the number of particles deposited on the alveolus wall from the first to the $N$ th breathing cycles to the total number of particles entering the alveolus during the $N$ breathing cycles. The time variation of deposition efficiency with the number of breathing cycles is shown in Figure 8. Accordingly, the deposition efficiency increases with the breathing cycles and saturates asymptotically to a constant value for each particle size. Here, for about ten cycles, the deposition efficiency curves for smaller particles seem to have reached their pick values. The reason for this trend is that, initially, there are no particles in the alveolus and it takes at least ten

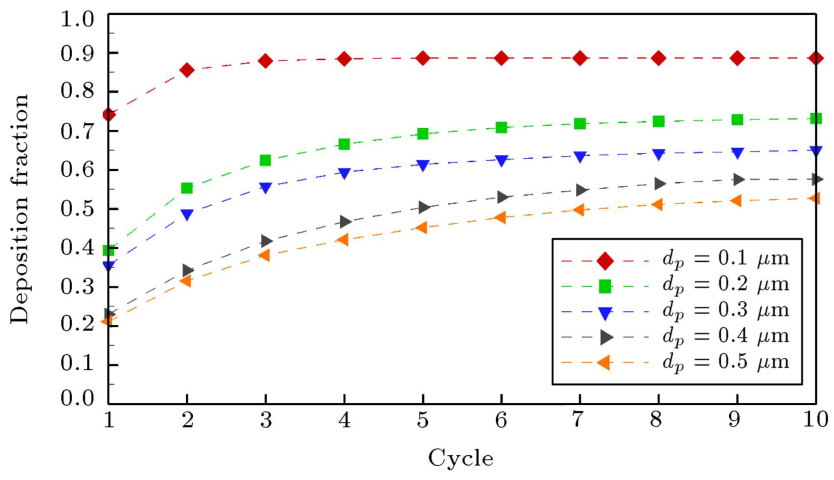

Figure 8. Variation of deposition efficiency during successive breathing cycles.

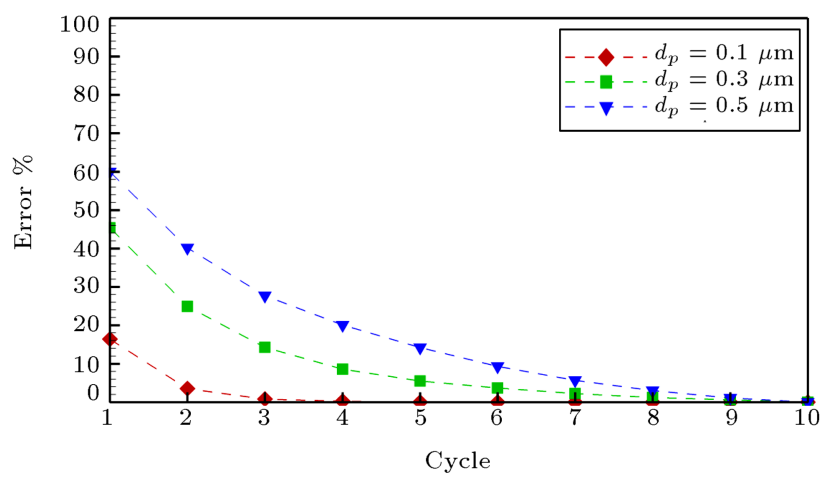

Figure 9. Errors in estimating steady-state deposition efficiency when considering only a few cycles in the simulation.

successive breathing cycles to generate a quasi-steady concentration in the alveolar cavity.

The computational errors for estimating the steady-state particle deposition (after ten cycles) are evaluated when just the first cycle is used and the results are shown in Figure 9. It can be seen that the error is more significant for larger particles, which have lower deposition efficiency and take longer to reach their steady value. For 0.5-micron particle, the error decreases by increasing the number of cycles from $60 \%$ in the first cycle to negligibly small in the 10th cycle.

\subsubsection{Comparison between uniform and parabolic radial distributions of particles}

To assess the importance of the injection of particles at the inlet, two sets of simulations with different inlet distributions were performed. In the first set, a uniform distribution of particles at the inlet is used and in the second set, a parabolic radial distribution of particles is injected at the inlet. The results for 0.3-micron particles are shown in Figure 10. It is seen that for the first set with uniform inlet distribution, the deposition efficiency is $33 \%$ higher than that for the second set with parabolic inlet distribution. This result can simply be explained by the fact that there is a low concentration of particles near the walls for 


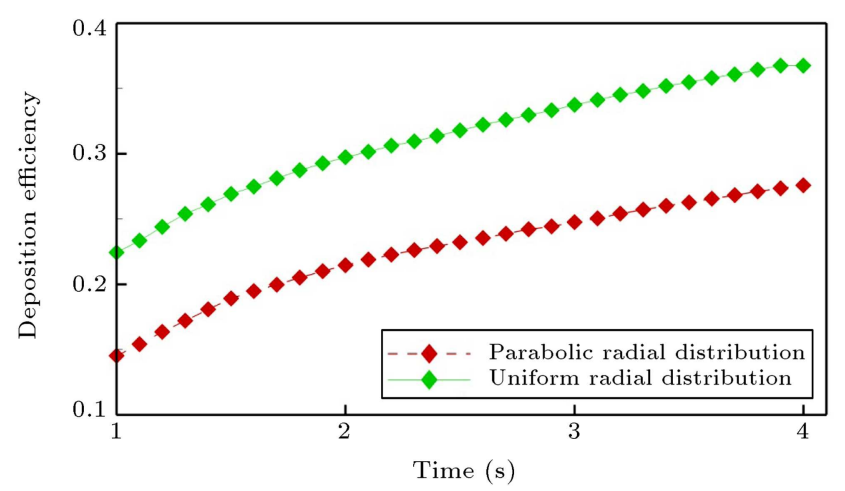

Figure 10. Comparison between parabolic and uniform radial distributions of 0.3 micron injected particles.

the parabolic inlet distribution compared with the uniform distribution. Therefore, the chance for particle deposition on the walls is higher for the uniform distribution than for the parabolic one.

\subsubsection{Comparison between pulse and continuous injection of particles}

In order to compare the effect of injection type on the deposition efficiency, two sets of simulations are performed. In the first set, particles are injected at the start of each cycle (pulse injection), while in the second set, they are incrementally injected during inhalation time in each breathing cycle (continuous injection). The results shown in Figure 11 indicate that in the first set, the deposition efficiency is about $4 \%$ higher than that in the second one. This observation can simply be explained by the fact that particles have more time to deposit on the walls when all are injected at the beginning of the inhalation cycle. Since the inhaled particles are drawn continuously through the lung in each breath, it is more realistic to simulate the continuous scenario instead of the all-at-once (pulse) particle injection one. The presented results show that the momentary injection, which has been used in previous literature, results in a slight overestimation of deposition efficiency.

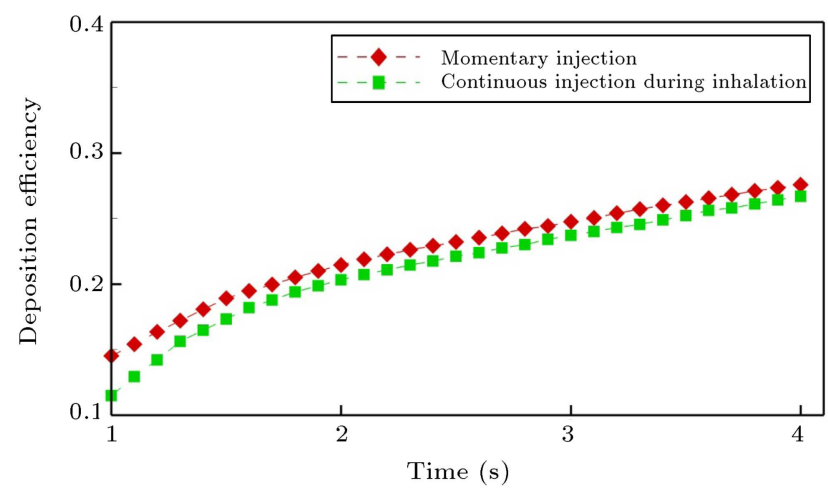

Figure 11. Comparison of deposition efficiencies for 0.3 micron particles predicted by pulse and continuous injection.

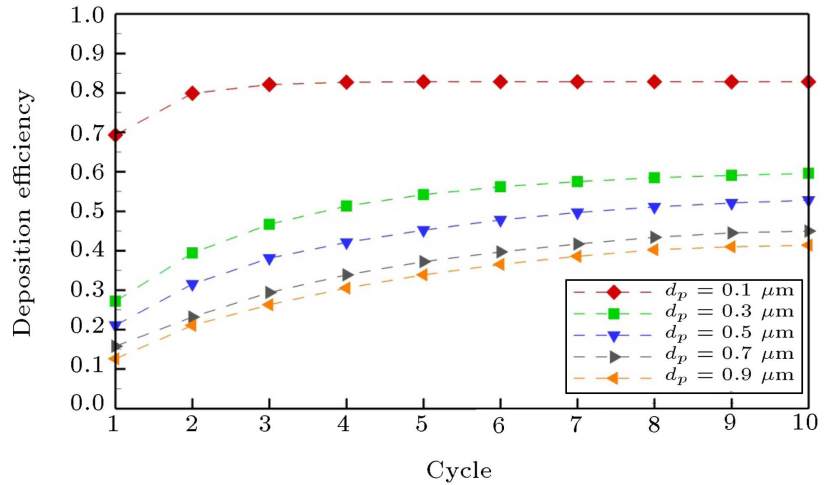

Figure 12. Deposition efficiency of submicron particles for ten successive breathing cycles.

\subsubsection{Deposition efficiency results}

As noted above, in order to simulate particle deposition in the alveolar cavity during breathing, the most realistic simulation conditions are successive breathing, parabolic radial distribution for injected particles, and continuous injection. The simulation results under these conditions are presented in Figure 12. Clearly, particles with smaller diameters have larger deposition efficiencies in the alveolus due to their increased Brownian motion.

\subsection{Normal gravity results}

When gravity is present, particles are also deposited by the sedimentation mechanism that could become important for particles in the micron range and larger. The predicted deposition efficiencies for gravity in different directions are presented in Figure 13 and compared with that in the zero-gravity condition. All values are calculated after 10 successive breadths.

According to Figure 13, the deposition efficiency increases when gravity acts in the $Z n$ and $Y p$ directions and decreases when gravity acts in the $Z p$ direction. These trends can be explained by the fact that gravity in the $Z n$ or $Y p$ direction accelerates the particles towards the alveolus wall and increases the deposition fraction. It qualitatively agrees with

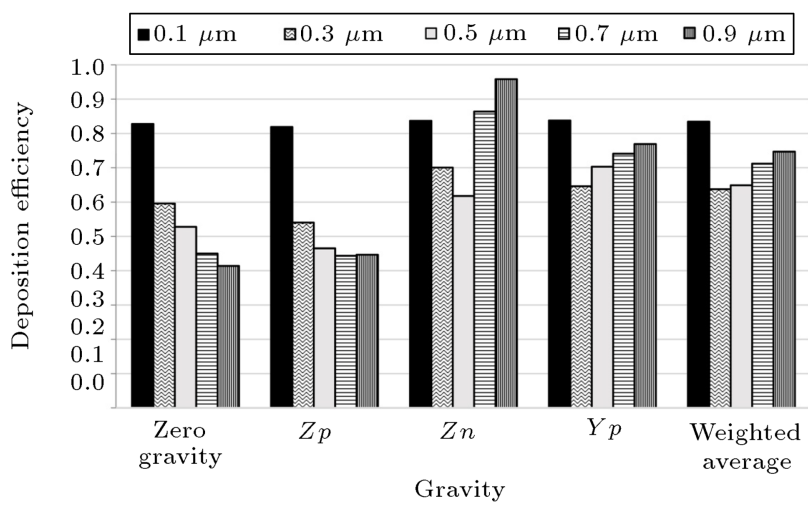

Figure 13. Deposition efficiency of submicron particles under zero and normal gravity conditions. 


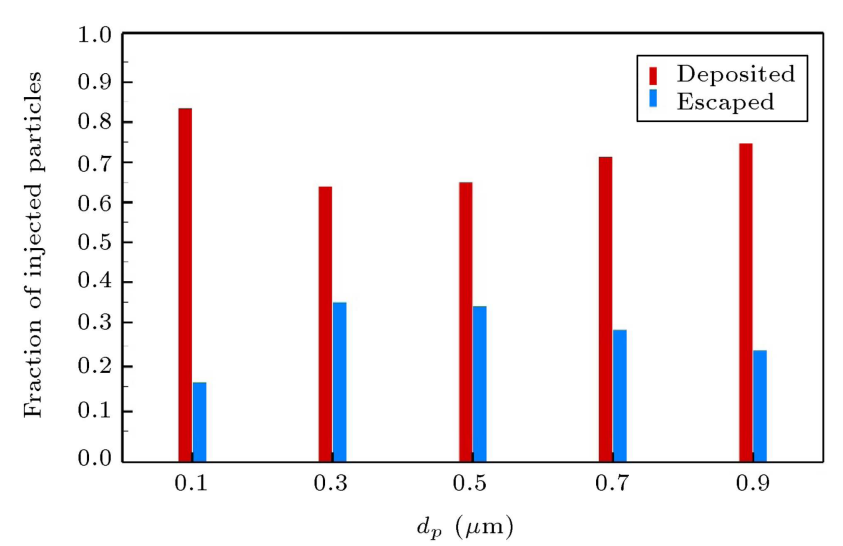

Figure 14. Deposited and escaped fractions of submicron particles under normal gravity condition with random orientation.

Ma and Darqueene results [11]. The larger particles have higher sedimentation velocities and their total deposition increases accordingly.

In Figure 14, the deposited and escaped fractions for particles with different sizes under normal gravity conditions, weighted averaged over different orientations of gravity, are shown. The deposition efficiency of smaller particles $(0.1 \mu \mathrm{m})$ and larger particles $(0.9 \mu \mathrm{m})$ is high due to their high Brownian diffusion and gravitational sedimentation, respectively. At the intermediate size of about $0.3 \mu \mathrm{m}$, the deposition efficiency has a mild minimum. Previous reports also predicted a minimal deposition for particles of about $0.3 \mu \mathrm{m}$ in diameter [24]. In this intermediate size, neither sedimentation nor Brownian diffusion is important compared with the small and large particle sizes; instead, escaped fraction is high. Thus, in order to select the optimum size of aerosol drugs with high deposition efficiency in alveolar region, this particle size is not appropriate. It is better to select smaller particles, which consequently have higher deposition due to their higher Brownian diffusion.

\subsection{Ultrafine particles deposition}

Ultrafine aerosols injected continuously during inspiration period with parabolic distribution have also been examined for deposition on the alveolus walls. For these small particles, gravity force is not significant; therefore, it is not included in the simulation. In contract to particles larger than $0.1 \mu \mathrm{m}$, deposition fraction of ultrafine particles quickly reaches steady state after the first or second cycle. The deposition efficiency results are shown in Figure 15 for 0.03, 0.05, 0.07 , and $0.09 \mu \mathrm{m}$ particles. It has been found that for ultrafine particles (smaller than $0.1 \mu \mathrm{m}$ ), deposition efficiency monotonically increases by decreasing the particle diameter. Nanoparticles have the highest deposition efficiency in the alveoli $(87 \%)$ due to their high diffusivity.

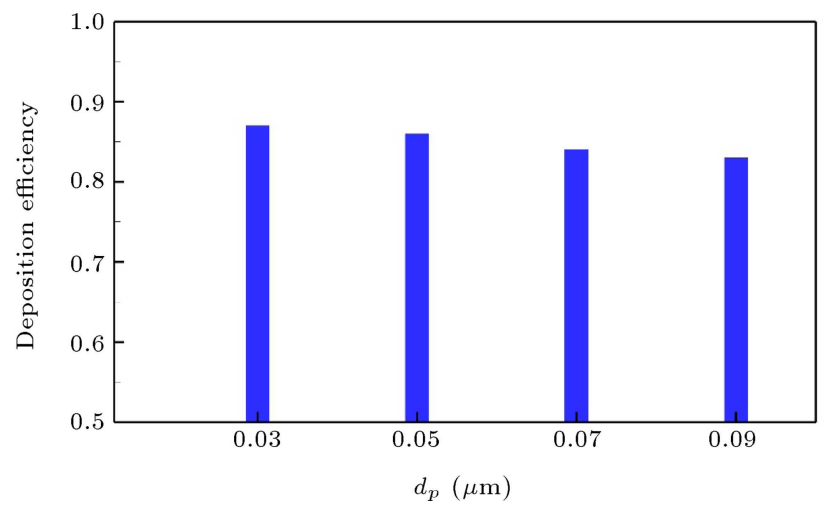

Figure 15. Deposition efficiency of nanoparticles.

\section{Discussion and limitations}

The problem for pulmonary delivery of large particles is their high oropharyngeal deposition, which gives local side effects. Although small particles $(\sim 1 \mu \mathrm{m})$ have a greater potential to be exhaled (about $10 \%$ of dose), this is counterbalanced by high pulmonary deposition and low oropharyngeal deposition [25]. Thus, submicron-size particles can penetrate deep into alveolar region of the lung.

Our findings show that alveolar deposition efficiency for submicron particles is high (higher than $60 \%$ ). These results seem to be different from the published experimental tests on human and animal [2628 ], in which smaller overall deposition fraction (lower than $30 \%$ ) has been measured for both total lung and alveolar region. The overall deposition fraction is defined as the amount of particles captured in the alveolar region compared to the amount of particles entering through the mouth or nose. Here, the deposition efficiency is evaluated for the particles that penetrate deep into the lung and enter the alveolar region. That is, the deposition efficiently is defined as the fraction of particles that are deposited in the alveolar region compared to that entering this region.

The presented results show that in the particle diameter range of 0.1 to $1 \mu \mathrm{m}, 0.1 \mu \mathrm{m}$ (in the studied range) has the highest deposition efficiency in the alveoli, which is because of its high diffusion coefficient. Therefore, it can be deduced that particles smaller than $0.1 \mu \mathrm{m}$ will have even higher deposition in the alveoli due to their high diffusivity. Ultrafine particles will be quickly deposited on the walls of alveoli. The maximum deposition efficiency ( $87 \%$ ) in the alveoli belongs to 30 $\mathrm{nm}$ particles. Ultrafine particles constitute the major concentration of airborne pollutants mainly emitted from motor vehicles, including lead and mercury. Small size and surface characteristics of these particles play an important role in toxicity. Also, they have slow clearance mechanism in the pulmonary region and can penetrate into interstitial region and translocate to other organs [29]. Ultrafine particles can be associated 
with a range of acute and chronic effects. These consequences may pose health risks to the exposed population.

The presented results may find application to aerosolized systemic drug delivery. Most of pulmonary drug delivery devices have been designed to produce particles in the range of $1-5 \mu \mathrm{m}$. For particles in the size range of 0.1 to $1 \mu \mathrm{m}$, neither sedimentation nor Brownian motion is strong enough to cause high deposition on extrathoracic and tracheobronchial regions. Thus, because of their low inertia, they may be easily exhaled following inspiration before they can reach the alveolar region. The challenge for delivery of ultrafine particles is related to their high deposition in the extrathoracic and tracheobronchial regions due to their high Brownian motion [24]. As a result, very few fine particles reach the alveolar airways to be available for deposition. In order to overcome this challenge, agglomerated vesicle and liposomal drug formulation for pulmonary drug delivery have been developed [30]. These vesicles are of multi-micron sized chemically linked agglomerates of core ultrafine particles. The particles are then released when the vesicle reaches the alveolar region and are deposited on the alveolar walls.

One limitation of the current study is using an idealized geometric model for alveolus. Using more realistic geometries from high-resolution micro CT images would improve the applicability of these simulations. It should be mentioned that the current simulation is mostly restricted to terminal alveolar sacs and further extensions could be performed to more proximal acinar airways and bifurcations. Another limitation of the present study is the lack of direct experimental validation of submicron-size particle transport in the alveoli. Also, in most previous modeling studies, micron-size particles have been used. However, our fluid and solid dynamics results are validated by other previous experimental or simulation works.

This study is also limited to normal breathing in healthy humans. Prediction of particle deposition for breathing pattern with the use of mechanical ventilators needs further studies.

\section{Conclusions}

A 3D model of deforming alveolus was developed, which could help in better understanding of alveolar mechanics, airflow and particle transport, and deposition processes. A series of Flow-Structure Interaction (FSI) simulations under realistic breathing cycles were performed. The airflow obtained showed a good agreement with earlier works. The flow stream lines confirmed that the flow was radial with no circulation region. The resulting airflow fields in the alveolus were imported in an in-house particle tracking routine. The corresponding particle deposition efficiencies for different diameters under different scenarios were evaluated. Under zero-gravity conditions, the smaller particles had higher deposition efficiency. The effects of successive breathing cycles, uniform or parabolic radial distribution of injected particles, pulse or continuous injection, and gravity on particle deposition fraction were investigated. The results showed that in order to find long-term adverse effects of air pollutant particulate as well as therapeutic strategies for aerosolized drug delivery, at least ten succeeding breathing cycles should be considered in the particle tracking simulations. Moreover, using a uniform inlet particle distribution would markedly overestimate the particle deposition efficiency. Since continuous injection is closer to breathing phenomenon, pulse injection would slightly overestimate the particle deposition efficiency. For larger particles, the increase in deposition efficiency by the influence of gravitational sedimentation would be more than that for smaller ones. Therefore, the plot of deposition efficiency versus particle diameter shows a minimum around $0.3 \mu \mathrm{m}$. Thus, the optimum size for aerosol drugs with high deposition efficiency in alveolar region is the smaller one, which consequently has higher deposition due to their higher Brownian diffusion. Based on the presented results, it may be concluded that the deposition efficiency of submicron particles in alveolar cavity is generally high. Particularly, aerosols of diameter $0.03-0.1 \mu \mathrm{m}$ are recommended for efficient drug deposition (higher than $80 \%$ ). Finally, the results suggest therapeutic aerosolized drug delivery using agglomerated vesicle technology to reach the particles into the alveoli.

\section{Nomenclature}

$\begin{array}{ll}\text { FSI } & \text { Fluid-Structure Interaction } \\ d^{f, s} & \begin{array}{l}\text { Displacement vector ( } f \text { denotes fluid, } s \\ \text { denotes solid) }\end{array} \\ G & \text { Reduced relaxation function } \\ n_{i} & \text { Normal vector } \\ p & \text { Fluid pressure } \\ v_{i} & \text { Velocity vector }\end{array}$

\section{Greek symbols}

$\begin{array}{ll}\delta_{i j} & \text { Kronecker delta } \\ \varepsilon & \text { Strain rate } \\ \lambda & \text { Stretch ratio } \\ \mu & \text { Dynamic viscosity of the fluid } \\ \sigma_{0} & \text { Instantaneous stress response } \\ \sigma_{i j}^{f, s} & \text { Cauchy stresses tensor ( } f \text { denotes fluid, } \\ & s \text { denotes solid) } \\ \rho_{f, s} & \begin{array}{l}\text { Material density }(f \text { denotes fluid, } s \\ \text { denotes solid) }\end{array}\end{array}$




\section{References}

1. Bennett, W.D., Brown, J.S., Zeman, K.L., Hu, S.C., Scheuch, G. and Sommerer, K. "Targeting delivery of aerosols to different lung regions", J. of Aerosol Med., 15, pp. 179-188 (2002).

2. Edwards, D.A. and Dunbar, C. "Bioengineering of therapeutic aerosols", Ann. Review of Biomed. Eng., 4, pp. 93-107 (2002).

3. Foral, P.A., Malesker, M.A., Huerta, G. and Hilleman, D.E. "Nebulized opioids use in COPD", CHEST, 125(2), pp. 691-694 (2004).

4. Bonner, J.C., Card, J.W. and Zeldin, D.C. "Nanoparticle-mediated drug delivery and pulmonary hypertension", Hypertension, 53(5), pp. 751-753 (2009).

5. Madl, P. and Hussain, M. "Lung deposition predictions of airborne particles and the emergence of contemporary diseases - Part II", The Health, 2(3), pp. 101-107 (2011).

6. Hofmann, W. "Modelling inhaled particle deposition in the human lung-A review", J. of Aerosol Sci, 42, pp. 693-724 (2011).

7. Hussain, M., Madl, P. and Khan, A. "Lung deposition predictions of airborne particles and the emergence of contemporary diseases- Part-I", The Health, 2(2), pp. 51-59 (2011).

8. Haber, S., Yitzhak, D. and Tsuda, A. "Gravitational deposition in a rhythmically expanding and contracting alveolus", J. of Appl. Physiol., 95, pp. 657-671 (2003).

9. Harrington, L., Kim Prisk, G. and Darquenne, C. "Importance of the bifurcation zone and branch orientation in simulated aerosol deposition in the alveolar zone of the human lung", J. of Aerosol Sci., 37(1), pp. 37-62 (2006).

10. Darquenne, C., Harrington, L. and Prisk, G.K. "Alveolar duct expansion greatly enhances aerosol deposition: a three-dimensional computational fluid dynamics study", Philosophical Transactions of the Royal Society, 367, pp. 2333-2346 (2009).

11. Ma, B. and Darquenne, C. "Aerosol deposition characteristics in distal acinar airways under cyclic breathing conditions", J. of Appl. Physiol., 110(5), pp. 12711282 (2011).

12. Henry, F.S., Haber, S., Haberthür, D., Filipovic, N., Milasinovic, D., Schittny, J.C. and Tsuda, A. "The simultaneous role of an alveolus as flow mixer and flow feeder for the deposition of inhaled submicron particles", J. of Biomech. Eng., 134, pp. 121001-1121001-11 (2012).

13. Dailey, H.L. and Ghadiali, S.N. "Fluid-structure analysis of microparticle transport in deformable pulmonary alveoli", J. of Aerosol Sci., 38(3), pp. 269-288 (2007).

14. Kumar, H., Tawhai, M.H., Hoffman, E.A. and Lin, CL. "The effects of geometry on airflow in the acinar region of the human lung", J. of Biomech., 42(11), pp. 1635-1642 (2009).
15. Sugihara, T., Martin, C.J. and Hilderbrandt, J. "Length-tension properties of alveolar wall in man", J. of Appl. Physiol., 30, pp. 875-878 (1971).

16. Denny, E. and Schroter, R.C. "Viscoelastic behavior of a lung alveolar duct model", J. of Biomech. Eng., 122(2), pp. 143-151 (2000).

17. Oakes, J.M., Day, S., Weinstein, S.J. and Robinson, R.J. "Flow field analysis in expanding healthy and emphysematous alveolar models using particle image velocimetry", ASME J. of Biomech. Eng., 132(2), 021008, pp. 1-9 (2010).

18. Li, A. and Ahmadi, G. "Dispersion and deposition of spherical particles from point sources in a turbulent channel flow", J. of Aerosol Sci. and Tech., 16, pp. 209-226 (1992).

19. Tsuda, A., Butler, J.P. and Fredberg, J.J. "Effects of alveolated duct structure on aerosol kinetics. I. Diffusional deposition in the absence of gravity", $J$. Appl. Physiol., 76(6), pp. 2497-509 (1994).

20. Sznitman, J., Heimsch, F., Heimsch, T., Rusch, D. and Rösgen, T. "Three-dimensional convective alveolar flow induced by rhythmic breathing motion of the pulmonary acinus", J. of Biomech. Eng., 129, pp. 658665 (2007).

21. Harding Jr., E.M. and Robinson, R.J. "Flow in a terminal alveolar sac model with expanding walls using computational fluid dynamics", J. of Inhalation Toxicology, 22(8), pp. 669-678 (2010).

22. Rausch, S.M.K., Haberthür, D., Stampanoni, M., Schittny, J.C. and Wall, W.A. "Local strain distribution in real three-dimensional alveolar geometries", Ann. Biomed. Eng., 39(11), pp. 2835-2843 (2011).

23. Monjezi, M. and Saidi, M.S. "Fluid-structure interaction analysis of airflow in pulmonary alveoli during normal breathing in healthy humans", Scientia Iranica, 32(4), pp. 1826-1836 (2016).

24. International Commission on Radiological Protection (ICRP), Human Respiratory Tract Model for Radiological Protection, Tarrytown, NY, Elsevier Science Ltd. (1994).

25. Leach, C.L., Davidson, P.J. and Boudreau, R.J. "Improved airway targeting with the CFC free HFAbeclomethasone metered-dose inhaler compared with CFC-beclomethasone", Eur. Respir. J., 12, pp. 13461353 (1998).

26. Heyder, J., Gebhart, J., Heigwer, G., Roth, C. and Stahlhofen, W. "Experimental studies of the total deposition of aerosol particles in the human respiratory tract", J. of Aerosol Sci., 4, pp. 191-208 (1973).

27. Cuddihy, R.G., Brownstein, D.G., Raabe, O.G. and Kanapilly, G.M. "Respiratory tract deposition of inhaled polydisperse aerosols in beagle dogs", $J$. of Aerosol Sci., 4, pp. 35-45 (1973). 
28. Wolff, R.K., Kanappily, G.M., DeNee, P.B. and McClellan, R.O. "Deposition of $0.1 \mu \mathrm{m}$ chain aggregate aerosols in beagle dogs", J. of Aerosol Sci., 12, pp. 119-129 (1981).

29. Hussain, M., Winker-Heil, R. and Hofmann, W. "Lung dosimetry for inhaled long-lived radionuclides and radon progeny", Radiat Prot Dosim., 145(2-3), pp. 213-217 (2011).

30. Bhavane, R., Karathanasis, E. and Annapragada, A.V. "Agglomerated vesicle technology: a new class of particles for controlled and modulated pulmonary drug delivery", J. of Controlled Release, 93(1), pp. 15-28 (2003).

\section{Biographies}

Mojdeh Monjezi is a PhD candidate at the Center of Excellence in Energy Conversion, School of Mechanical Engineering, Sharif University of Technology (SUT). She received her BSc and MSc degrees from Isfahan University of Technology, Isfahan, Iran. Her research interests are biofluid simulation, modeling of particle transport and respiratory systems, and aerosol dynamics.

Mohammad Said Saidi is a Professor of Mechanical Engineering at Sharif University of Technology. His research interests are modeling and numerical analysis of transport and deposition of aerosol particles, modeling and numerical analysis of biofluids, and modeling and numerical analysis of thermal-hydraulics of porous media and microchannels.

Goodarz Ahmadi is a Professor of Mechanical and Aeronautical Engineering at Clarkson University. He has been awarded the title of "Clarkson Distinguished Professor". Some of his research interests include multiphase and granular flows, three-phase slurry flows, aerosols, micro-contamination control, turbulence modeling, stability of fluid motions, continuum mechanics, nonlinear random vibrations, and earthquake engineering. 